\title{
Virtual Power Plant Platform for Demand Response Based on Microservice Architecture
}

\author{
Guodong JIANG ${ }^{\mathrm{a}, 1}$, Zuixi XIE ${ }^{\mathrm{b}}$, Tao HAN ${ }^{\mathrm{a}}$, Hongwei DU ${ }^{\mathrm{a}}$, Rongqiang FENG $^{\mathrm{a}}$, \\ Shouquan $\mathrm{LUO}^{\mathrm{c}}$, Xueqiong $\mathrm{WU}^{\mathrm{a}}$, Jiaqi $Z \mathrm{ZHANG}^{\mathrm{a}}$ \\ ${ }^{a}$ NARI TECHNOLOGY CO., LTD, Nanjing 211106, China \\ ${ }^{b}$ Three Gorges Electric Energy (Guangdong) Co., Ltd, Shenzhen 518000, China \\ ${ }^{c}$ State Grid Electric Power Research Institute, Nanjing 211106, China
}

\begin{abstract}
It is a trend to use virtual power plant technology to realize demand response and participate in electricity trading. We design and implement the software control platform of virtual power plant for demand response. For this software platform, we analysed the requirements and got the overall architecture of the platform. On this basis, we design and implement the microservice architecture, interface design, basic application function design, advanced application function design, hardware architecture, communication architecture and security encryption of the platform. Finally, we summarize the application of the platform, and put forward the direction of further research and development.
\end{abstract}

Keywords. virtual power plant, demand response, microservice, function design, communication architecture, security encryption

\section{Introduction}

Virtual power plant (VPP) is the aggregation of a variety of distributed energy resources (DER). Through advanced control, communication and metering technology, it can aggregate distributed generation, energy storage, flexible load and other adjustable resources, participate in the dispatching of power grid as a whole. It makes full use of the coordination and complementarity of DER's regulating characteristics to realize the reasonable optimal allocation and utilization of resources. As a special power plant, a virtual power plant presents the overall function and effect of a traditional power plant. It can be controlled and managed like a traditional power plant. It can submit power generation plans to the power grid, participate in the electricity market, implement peak load regulation and frequency regulation and other auxiliary services. As an energy management system, internal energy management of VPP has multiple functions such as self-coordination, self-management and self-control [1].

${ }^{1}$ Corresponding author: Guodong Jiang, NARI TECHNOLOGY CO., LTD, Nanjing 211106, China; Email: jiangguodong@sgepri.sgcc.com.cn. 
Distributed energy resources involved in demand response include distributed generation, air conditioning load, distributed energy storage, electric vehicle charging pile, etc. Many DERs are small in scale, large in number and scattered in dispersive layout. Due to legal and technical reasons, traditional demand response technology cannot use these resources. At the same time, the traditional demand response has low automation level, low accuracy, poor reliability, and the time scale of load regulation response is long, which is difficult to meet the timeliness requirements of power system safe operation $[2,3]$.

The virtual power plant is used to participate in the demand response, so that all kinds of users, especially small and medium capacity users, can participate in the demand response through the virtual power plant to obtain additional economic benefits; For power grid companies, it is beneficial to utilize small-scale flexible load resources, improve economic benefits and reduce construction investment; For the nation and society, the construction of virtual power plant can significantly reduce the combustion of fossil fuels and greenhouse gas emissions, promote the efficient and energy-saving operation of power grid, and effectively promote the optimization and transformation process of power grid [4].

This paper describes a virtual power plant platform for demand response, which is a flexible and extensible system. Demand response business has two characteristics: rapidly changing of functions and rapid altering of customers. The platform adopts microservice architecture, which has the advantages of loose coupling, high cohesion, good interface adaptability, high security and high reliability. So, it can meet the requirements of demand response. This platform can realize the scalability and high availability of the system for customers to join and exit at any time; For the rapid changing of functions, it can realize rapid development and rapid deployment. We analyzed the requirements of the software platform and designed the overall architecture, software architecture and hardware architecture; the software function and system interface are designed; The communication architecture and security architecture are described.

\section{Requirement Analysis}

Based on the requirements and current situation of Chinese power system, the VPP platform monitors and analyses DERs and traditional power, including traditional power, DG, controllable load, energy storage, etc. After that, the VPP platform sends the calculated instructions to the dispatching system D5000 and the load control system, and these two systems send the instructions to the AGC and AVC modules of the dispatching system after calculation. After its calculation, AGC and AVC modules send instructions to their DERs for execution. The centralized control platform of VPP does not directly send control instructions to DERs. These are shown in Figure 1. 


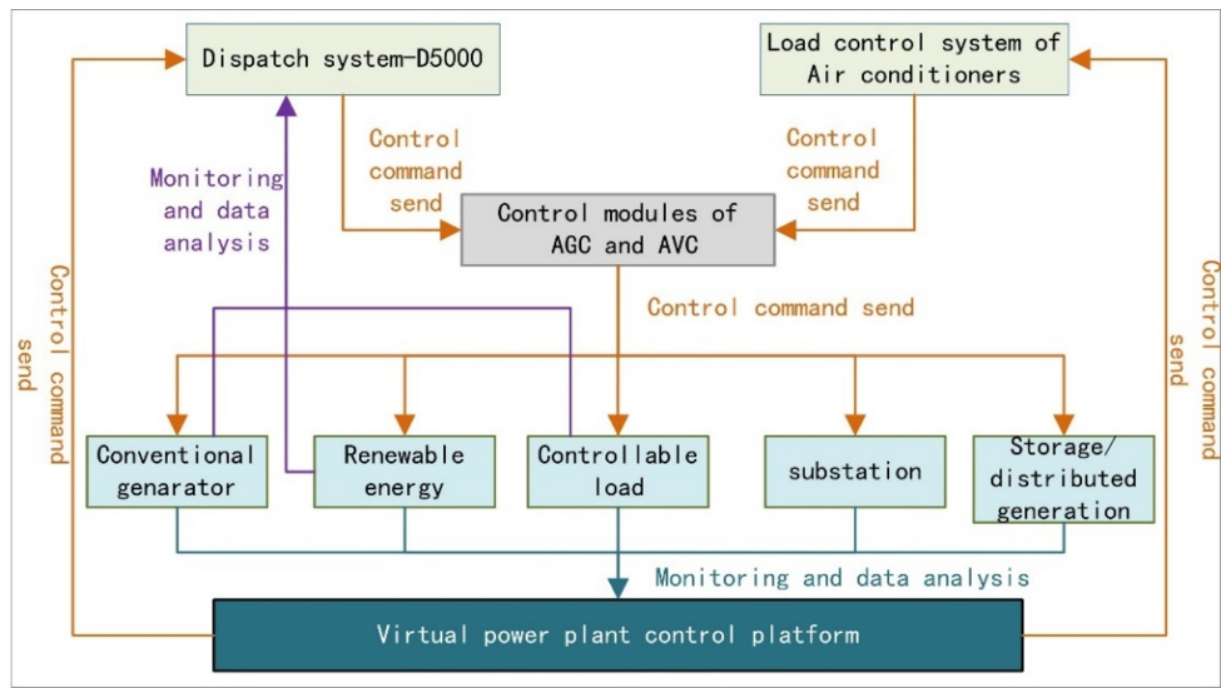

Figure 1. Virtual power plant platform diagram.

The VPP participates in the electricity market of demand response as an independent identity, and the following process is shown in Figure 2. Firstly, the power dispatching center releases the market demand, and then the VPP center interacts with it, including quotation, load control, settlement and so on. After the VPP platform receives the task, it decomposes and sends it to the end users, and the end users execute it. The VPP cooperates with the power grid dispatching center to complete the peak load regulation task, and makes settlement with the trading center.

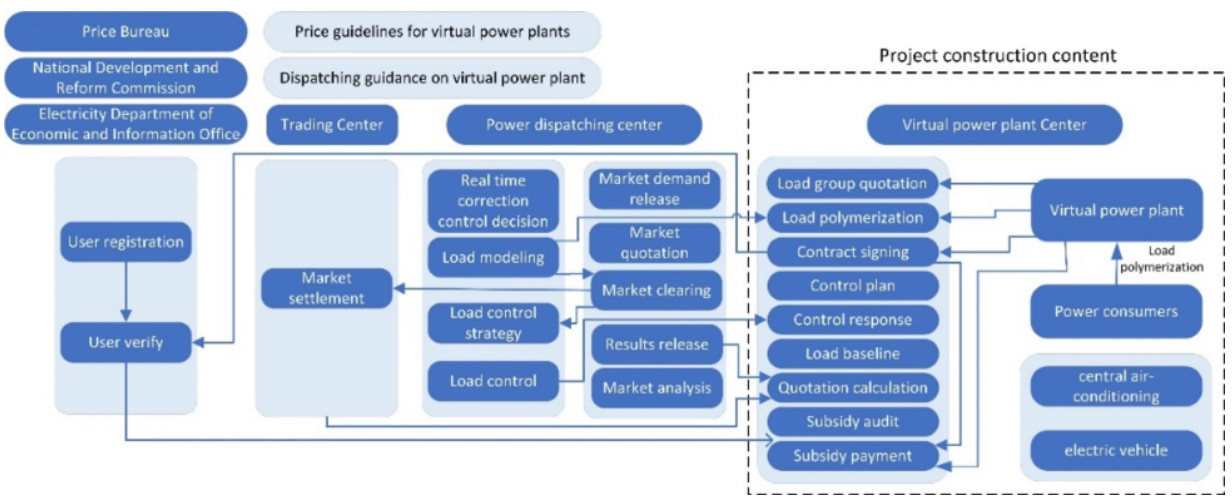

Figure 2. Operation flow chart of virtual power plant.

In order to complete this project, we have done the following three aspects.

One is to aggregate and evaluate the potential of DERs (mainly including air conditioning, small PV, etc.) added to the VPP. The aggregation parameters include the maximum and minimum power generation capacity (operating power constraints, cost and other indicators), the ramp rate of the VPP in each period, and various types of auxiliary service capacity (reserve capacity, power consumption, Cost and performance indicators etc. of the VPP).

The second is to establish a hierarchical, partitioned and multi-level dispatching and operation control architecture of VPP. Based on this framework, the multi-scale spatial- 
temporal fluctuation characteristics of DER output are analyzed, and the control strategy of power balance and operation mode optimization is proposed. The VPP collaborative interactive operation control technology is adopted.

The third is to design and implement the overall architecture of the virtual power plant platform, including process design, hardware architecture, software architecture, interface design, etc. We connect the VPP with the dispatch platform D5000 and the load control system, so as to realize the execution of the dispatch platform D5000 instructions and the optimal control of the internal DERs.

\section{Virtual Power Plant System}

The system diagram of VPP is shown in Figure 3. The operation architecture of VPP is shown in the figure. According to the mode of unified management, distributed access and collaborative control management, based on the power Internet of Things, the VPP system is constructed through intelligent edge computing, perception terminal, data intelligent fusion analysis, auxiliary management decision and other technical methods [5].

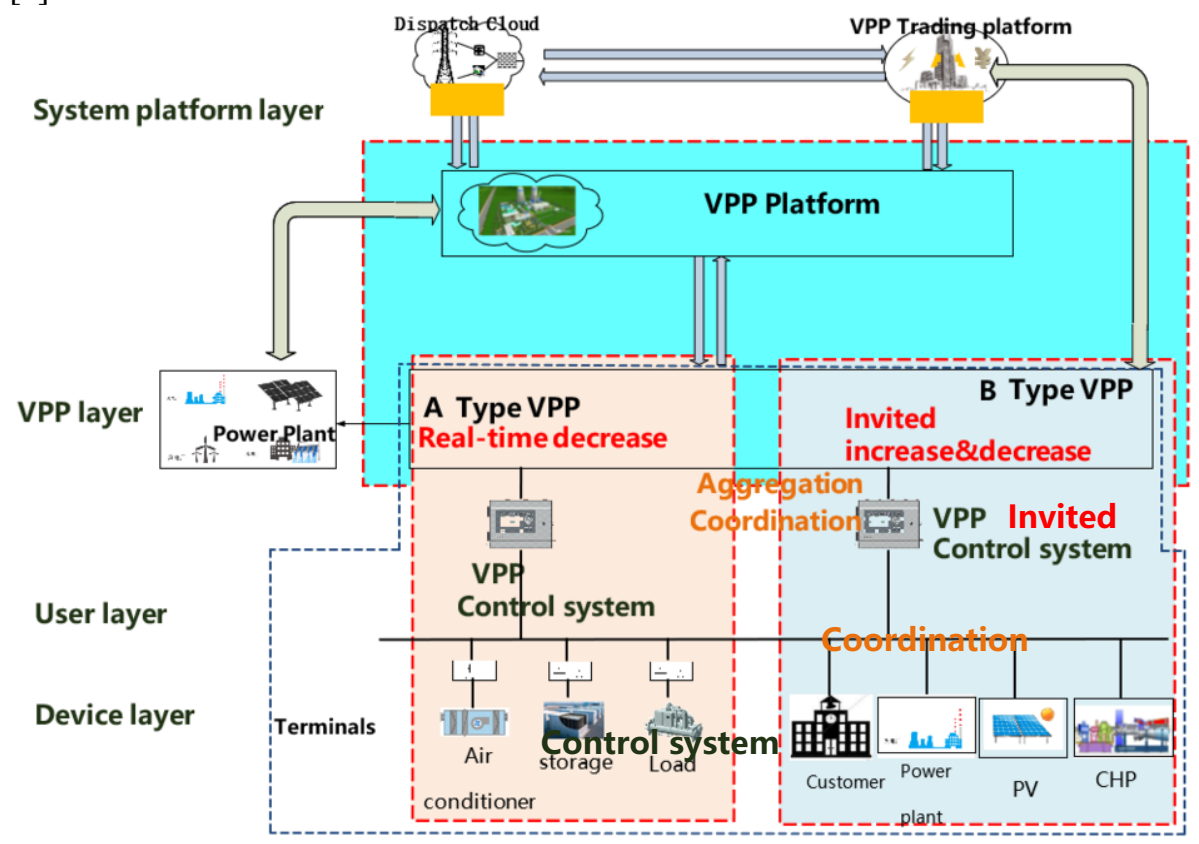

Figure 3. The system diagram of VPP.

The VPP system realizes the partition clustering of DERs and forms the external characteristics similar to traditional power plants. Then, the registration information, quotation information and contract information are submitted to the VPP platform. The VPP platform is responsible for reviewing the VPP access conditions, access scheme, external characteristics of the VPP, VPP files and response information, receiving dispatching cloud call information and reporting response information.

Relying on the existing unified power market trading platform, the VPP trading platform receives the dispatching requirements issued by the dispatching cloud, the access audit results submitted by the VPP platform, the external characteristics of the 
VPP, and the implementation identification results. Combined with the registration, quotation, and contract information reported by the VPP platform, the VPP trading platform carries out market clearing, and at the same time reports release clearing results to the VPP's every entity, VPP platform and dispatching cloud.

The VPP system divides DERs into class A and class B. Class A VPP mainly include industrial and commercial residential building air conditioning, user side energy storage and industrial user load. These objects are adjusted and reduced in real time, and participate in the transaction of power grid peak shaving auxiliary services. Class B VPP are mainly large users, self-provided power plants, distributed photovoltaic power plants and triple generation power plants. They are invited to increase or decrease these kinds of objects and participate in the auxiliary service transaction of peak load cutting and valley filling.

VPP system is divided into four layers, namely Device layer, User layer, VPP layer and System platform layer.

Device layer: the VPP resources on the demand side. The project considers the load of building central air conditioning and split air conditioning and so on.

User layer: air conditioning intelligent control terminal is deployed in public buildings, so that the VPP layer can connect with it to control demand side resources.

VPP layer: build virtual unit control system. Monitoring and controlling the operation of VPP resources. It is a centralized control mode with flexible control resources. The VPP resources are actively aggregated, and the end users transfer the control right of resources to them, so that the VPP can control the demand side resources through price or other signals.

System platform layer: build VPP platform. There are several VPP units under the jurisdiction of VPP platform, and there is a decentralized control mode between VPP platform and VPP units. Its instructions include the upload and release of instructions and data monitoring. The VPP platform directly participates in the power market transactions and receives the control cloud response instructions.

\section{Software Design}

\subsection{Microservice Architecture}

Microservice architecture decouples solutions by decomposing functions into several services. By splitting a large application and service into several or even dozens of microservices, the microservice architecture implements the extension of a single component rather than the stack of the whole application. In this way, developers can create applications around a single business domain component, and develop, manage and iterate these applications independently, which greatly improves the scalability. In the distributed components, by Using Cloud Architecture and platform deployment, the delivery and management of products become easier [6].

Compared with traditional monolithic architecture, micro service has the following advantages: project can be delivered continuously, service can be expanded independently, compatibility is stronger, technology upgrading and simultaneous interpreting are quicker and more convenient. 
Microservice architecture can effectively split applications, realize agile development and deployment, and solve the problems of traditional monolithic architecture such as tight coupling, inflexible deployment and poor scalability. Microservice architecture is a distributed overall solution, including service registration and discovery, service configuration, link monitoring, service gateway, load balancing, service fusing and other functions. This project selects the microservice framework spring cloud, and the architecture diagram is shown in Figure 4.

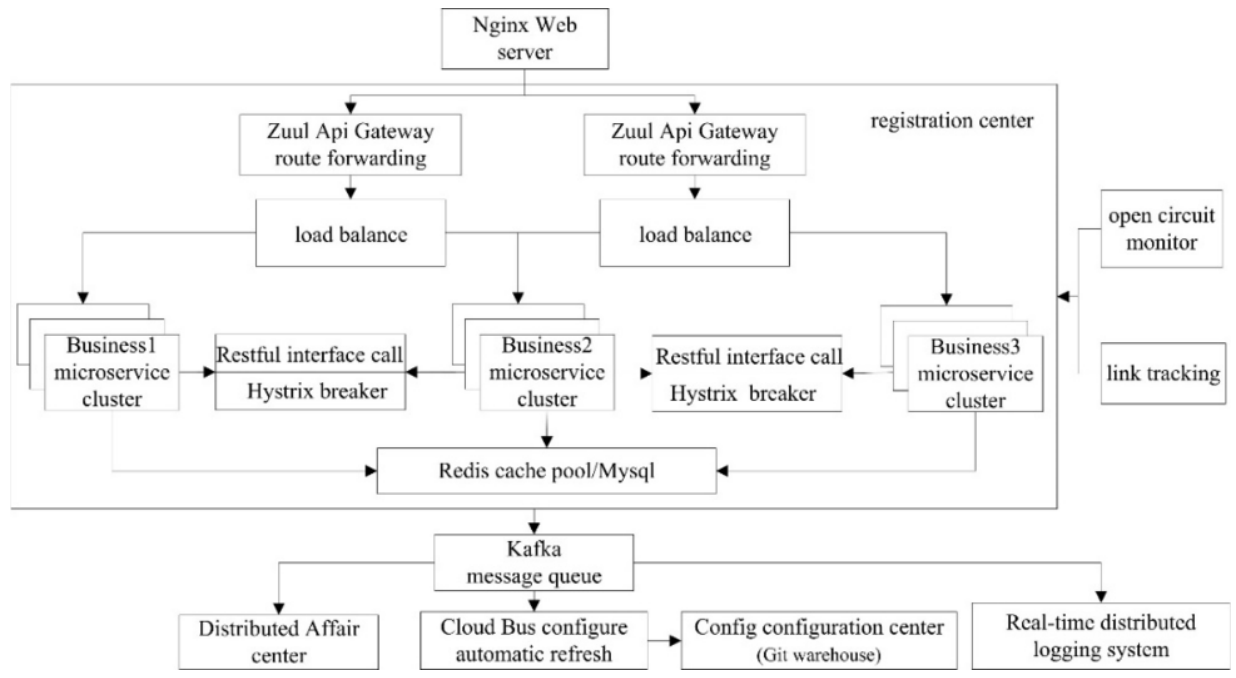

Figure 4. Overall architecture of microservice.

The running process of microservice architecture is: firstly, all microservices are deployed to each server and registered in the registry. When the front end sends an HTTP or HTTPs request to the Nginx server, the Nginx server checks its identity. After the verification is passed, Nginx forwards the request to a micro service in the registry through the router.

Microservices can call each other. When Microservice A calls Microservice B, it needs to obtain the registered address and port of Microservice B from the microservice registry. Since Microservice B may have multiple copies, it needs to select one of the interfaces exposed by the copy service of Microservice B to call through the load balancing polling mechanism. The breaker mechanism is introduced in the process of microservice call to avoid the microservice call exception caused by a microservice failure, which leads to the whole microservice crash. In this way, when one of the micro services fails, other micro services can run stably [7].

Redis is used to cache real-time data, and MySQL relational database is used to store static data and historical data. Microservices decouple the call relationship among microservices through Kafka message queue. The architecture can change the characteristics of microservices through configuration parameters, and manage and maintain the configuration information of each microservice through the configuration center. It can monitor the running of microservice in real time. Restful API interface is used when the third-party system wants to call its own microservices or the microservices inside the system call each other. 


\subsection{Platform Interface Design}

This work mainly includes the interface design of the third-party system and data acquisition. The interface design is shown in Figure 5:

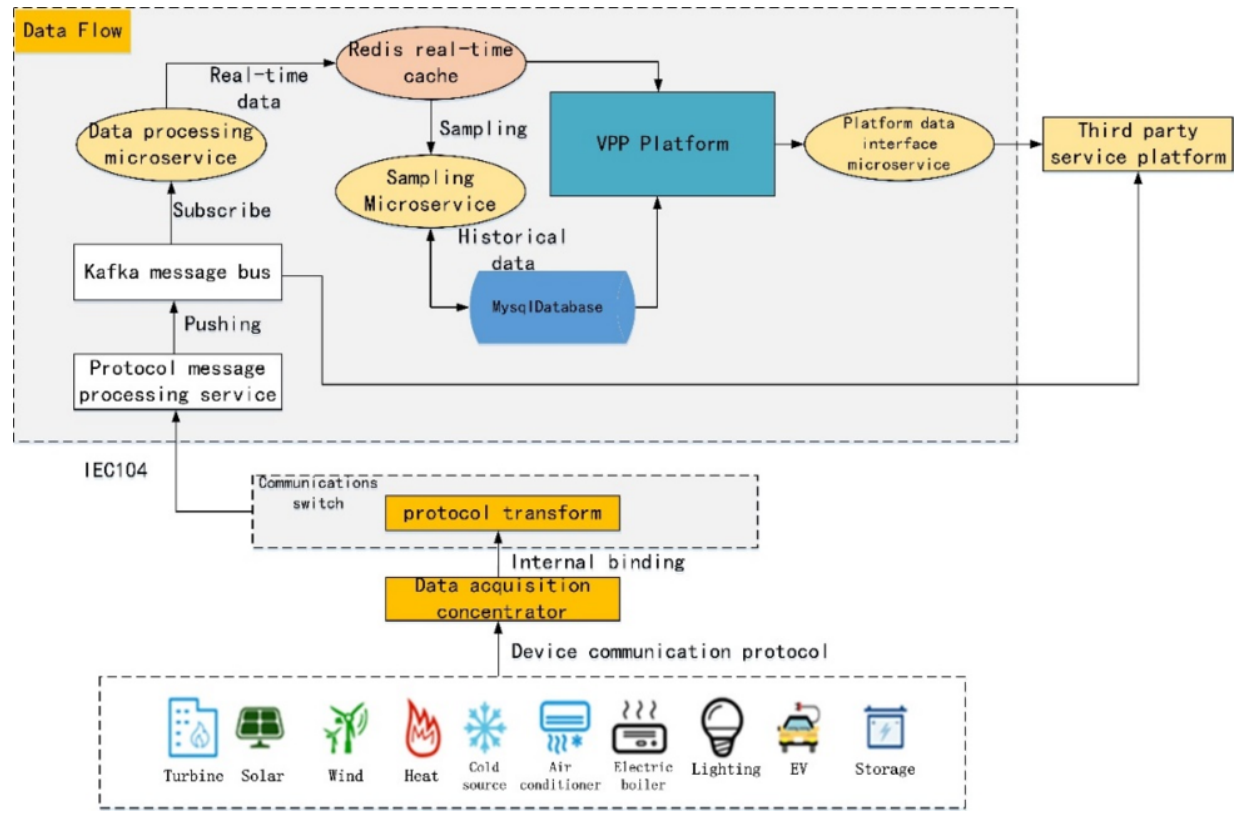

Figure 5. Platform Interface Design Drawing

The data collection of various energy equipment is connected to the platform through IEC104 protocol. After the protocol processing program processes the mature data, it pushes the mature data into the related topics of Kafka message bus. The microservice subscribing to this topic obtains the data for real-time analysis, stores the real-time data into the real-time database Redis, and the sampling micro service samples the real-time data and writes it into the history database MySQL. By defining the threeparty interface microservice, real-time data, static data and historical data are obtained from Redis and MySQL, and the data is released to the third-party system through the standard restful interface.

\subsection{Software Function Design}

The software architecture of VPP platform is divided into four layers, namely hardware layer, platform layer, basic applications layer and advanced applications layer [8]. The software architecture is shown in Figure 6. 


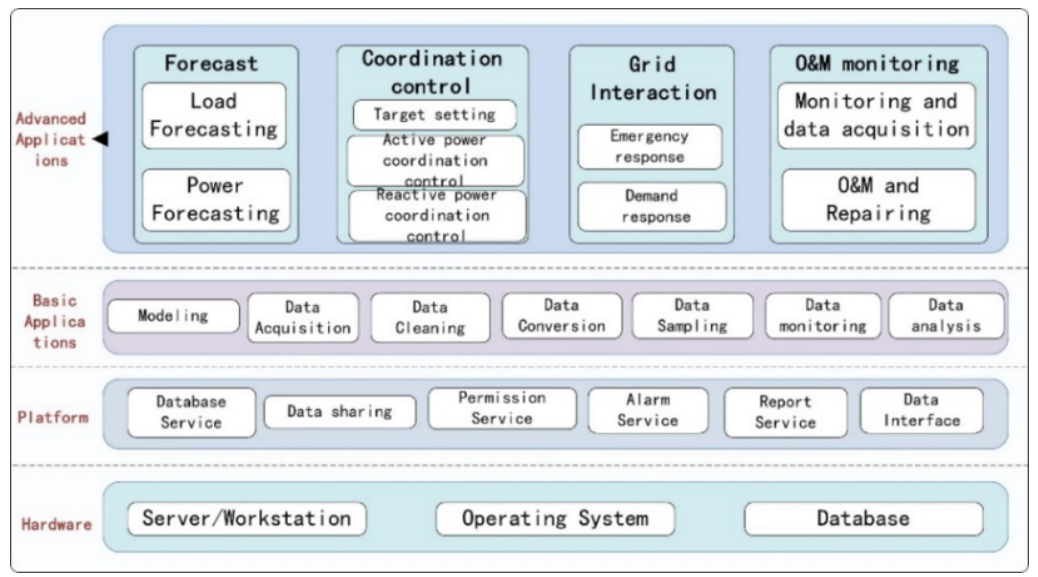

Figure 6. Software architecture of VPP platform.

\subsubsection{Basic Application Functions}

\section{Modelling}

It has a model covering the whole business area of VPP and meets the following functional requirements:

(1) According to IEC61850, IEC61968, IEC61970 and other standards and specifications, a complete set of object model is constructed.

(2) It has system hierarchical organization model object.

(3) Equipment model object with system control and analysis.

(4) Network model objects with physical associations.

(5) Business model objects with logic.

\section{Data acquisition}

The communication mode of data acquisition and control can adopt serial communication, twisted pair communication, optical fiber communication and wireless communication, and the communication protocol can adopt Modbus, DL / t634.5101, DL / t634.5104, etc. Ethernet is used to communicate with grid dispatching automation system and load centralized control system. IEC104, Webservice and HTTP are used as communication protocols.

The acquisition of real-time operation information mainly includes but is not limited to the following contents:

(1) Remote data of power generation, heating, cooling and gas supply monitoring system. etc.

(2) Operation data of power grid, cooling / heating network, gas supply network,

(3) Electric, heat, cold, gas and other load operation data.

(4) Operation data of power storage, heat storage, cold storage, gas storage, charging pile and other equipment or systems.

(5) Electricity, heat, cold and gas metering information.

(6) Data acquisition of temperature, pressure, flow, heat and valve opening in heat exchange station.

(7) Power quality data.

(8) Meteorological and environmental monitoring data.

(9) Other system data. 


\section{Data cleaning}

Data cleaning is to solve the problem of data quality and make data more suitable for mining. The result of data cleaning is to deal with all kinds of disordered data and get standard, high-quality and continuous data, which provides data sources for big data statistics and big data mining. The main functions include:

(1) Identify the integrity of supplementary data

(2) Screening the uniqueness of data

(3) Study and judge the legitimacy of data.

\section{Data conversion}

Data conversion is modeling based on device container, which provides support for multi-source big data standardization collection. No matter what equipment, system, protocol and communication mode are connected, the integrated energy management and control platform can be easily connected, and all data collection tasks can be completed in one data acquisition system, it is convenient for model synchronization between different platforms, realizing the same function processing logic and providing the same external services, so as to realize the unified operation, maintenance and display of different collection tasks. Be different from the conventional database architecture change, data conversion can adapt to the increasing amount of data, the original data architecture is unreasonable, cannot meet the requirements of all aspects, from the replacement of the database, the replacement of the data structure, into the conversion of the data itself. The main functions are as follows:

(1) Support a variety of communication methods (analog, digital, dial-up, network) and communication structure (point-to-point, multi-point collinear, star, ring).

(2) It supports online extension protocol types, provides standard protocols such as IEC60870-5-101 and IEC60870-5-104, supports industrial control communication protocols such as MODBUS, and also supports user-defined protocols. For authorized users, the specification library can be expanded by API functions provided by the system.

(3) In addition to conventional measurement data, data types are also compatible with unstructured data such as pictures and video streams.

(4) It supports wired and wireless communication; It supports two logical communication modes: fixed IP terminal and dynamic IP terminal.

(5) Multi security mechanism: it has multiple security mechanisms, such as dual standby, multi machine switching, data encryption, etc.

(6) Provide a series of convenient and practical data query interface. Including: hardware interface configuration, channel code monitoring, various types of data monitoring, device status monitoring, bit error rate monitoring, database configuration.

\section{Data sampling}

According to the massive data types sent by the front-end acquisition server, such as alarm information, video information, remote control information, analog quantity, state quantity, etc., the platform classifies them according to the data types, provides different optimization processes of data writing to the database, and writes the data to the real-time database or historical database respectively. To maximize the processing capacity of data server and application server. After the data is stored in the database, the platform provides data sampling and reading cycles according to real-time data and nonreal-time data: historical data provides sampling cycles ranging from 5 minutes, 10 minutes, 15 minutes, 20 minutes, 30 minutes and 60 minutes. Real time data provides a sampling period of 5 seconds ( Once the alarm information, switch position and protection status change, they will be read immediately.) The main functions are as follows: 
(1) Provide massive data writing

(2) Provide data sampling and reading period ranging from 1 second to 60 minutes

(3) Provide data warehousing correctness detection

(4) It provides integrated association of graph, model and library, and generates graph from library and library from graph.

(5) Provide database read permission by class-table-domain.

\section{Data monitoring}

On the one hand, data monitoring monitors the broadcast message and point-to-point message receiving sending transmission status in the process of data transmission, on the other hand, it monitors the network traffic and system resources caused by data transmission. The main functions are as follows:

(1) Provide process status query, log query, message sending and message receiving query.

(2) Provide network operation condition monitoring and network flow control.

(3) Provide system resource monitoring alarm, including CPU, memory, hard disk monitoring.

(4) Provide logging function, system exception log, system operation log, application operation log, etc.

\section{Data analysis}

The total power, electricity consumption and sales electricity of VPP and internal virtual units are analyzed; It makes a comparative analysis of ranking and comparison from the aspects of the trend of electricity, the nature of electricity consumption, the type of industry and the composition of electricity consumption.

\subsubsection{Advanced Application Functions}

\section{Load forecasting}

Load forecasting of VPP includes forecasting energy consumption, maximum load, minimum load, and the time interval of maximum load and minimum load. When drawing hourly load curves of typical days in different seasons, it should be superimposed hour by hour according to the type and nature of each load.

(1) Combined with historical data, weather, temperature and other factors, the basic operation status of load resources is predicted;

(2) Understand load resource control variables, control methods, influencing factors and customer comfort requirements;

(3) Based on the basic operation state of load resources, load regulation methods and comfort requirements, the adjustable potential of load resources is analyzed [9].

\section{Generation forecasting}

Through the identification, correction and similar day clustering of historical meteorological and wind power, photovoltaic, other power generation data, the dynamic optimal historical data set is established. The prediction results of different prediction algorithms under different classification clusters are analyzed and compared, and the adaptive optimal weighted combination generation prediction model is established. Realize the short-term power generation prediction of virtual power plant, and provide the short-term prediction results with a time resolution of 15 minutes in the next 1 to 3 days. 


\section{Coordination control}

Considering the security and stability constraints of power grid, load fluctuation and uncertainty of distributed generation output, the optimization mathematical model of VPP is established to maximize the economic, environmental and social benefits. It has the generation power prediction technology of distributed generation in VPP, focusing on the impact of climate conditions on the output of distributed generation.

\section{Operation target setting}

Operation objectives include two levels: power plant level operation objectives and component level operation objectives. The operation objectives of power plant level refer to the safe operation objectives of the units that can be controlled and regulated by the regional dispatching decision-making level, and the rated power and current of the microgrid; Component level operation target refers to the safe operation target of terminal components in virtual power plant, which generally includes the cut in and cut out wind speed of wind turbine, the rated capacity of photovoltaic inverter, etc. The system provides a friendly running target setting tool, which can be directly called to modify on the screen, and the user authority will be checked before the modification operation.

Through the distributed controllable units such as energy storage, photovoltaic, wind energy and load in the VPP, the stable operation of various modes such as economic mode, high reliability mode and isolated network mode can be realized, and the system can make automatic decision and control.

Active power coordination control

According to the real-time operation status, generation power prediction results, load prediction results and other information of the distribution network, under the constraints of system operation, the real-time optimal dispatching adjustment and control strategy are given for the active power output of each distributed energy in the VPP system, so as to improve the economy of real-time operation of the system.

According to the actual operation status of DGs and load, the power control strategy of each period in the future dispatching cycle is formulated to form a generation dispatching plan in the dispatching cycle, which is distributed to the controllers of each DER in the VPP system for execution. The objectives of switching power control, smooth output control and economic optimization operation can be realized respectively, the operation control mode can be switched manually or automatically according to the real-time operation status [10].

\section{Reactive power coordination control}

Control the system voltage to ensure that the system voltage operates in a safe area, and ensure the safety and reliability of the system operation.

The intermittent power supply (such as photovoltaic, wind power generation, etc.) in VPP system will cause voltage fluctuation at the grid connection point. The system analyses and calculates the data of new energy power generation unit through data acquisition, and then executes the command through output control to adjust / switch the equipment with reactive power regulation ability, including inverter, so as to make the output voltage and reactive power within the qualified range, so as to ensure the purpose of power quality. The reactive power control strategy determines the voltage control of the parallel node and the reactive power control of the inverter. 


\section{Emergency response}

In case of an accident, the adjustable resources in the VPP area can be controlled urgently to respond to the demand of frequency and voltage regulation of large power grid. According to its own operation state, by adjusting the adjustable resources, the DERs in the VPP is used as the power source to transmit power to the superior power grid. At the same time, the actual energy contribution of the system is counted, and the adjustable margin and the duration of emergency support are estimated.

\section{Demand response}

According to the instructions given by the superior power grid in advance, the VPP can respond to the invitation. The instructions issued in advance include specific indicators (demand response capacity, price, revenue, system operation trend, etc.) and invitation time range. In the specified time range, by adjusting the adjustable resources (flexible load, energy storage system, river water source system, etc.), the required operation indexes and related real-time operation trend are obtained.

\section{Monitoring information}

(1) The data management module collects the monitoring information after cleaning, mainly the operating parameters of VPP equipment, taking air conditioning as an example, including the power of the refrigerator, the temperature of the chilled water inlet and outlet water, etc;

(2) Organize and backup the monitoring information;

(3) The display method includes the platform interface display and web display of VPP. The operators of VPP can perform relevant operations in the display interface to realize information interaction;

(4) Meanwhile, the monitoring information is transmitted to the user, including web, app and other forms, so that users can grasp the operation of the equipment.

\section{Operation and maintenance repair}

Operation and maintenance control module includes business functions such as fault repair, equipment inspection, energy use application, planned maintenance and defect management. In view of the characteristics of various business types and processing processes, the operation and maintenance management module provide public service functions such as application approval, work order processing, stop supply plan / event, process configuration management, and flexibly supports business differentiation processing, Meet the business management needs of different users. In addition, the status monitoring and early warning of each business processing process are realized through alarm service, and the equipment operation monitoring and on-site repair processing are realized in mobile terminal.

\section{Communication Architecture}

This platform supports DL/T 698.45-2017 protocol (for China electric power industry), DL/T 645, CJ/T, MODBUS and other protocols [11]. The communication architecture is shown in Figure 7. 


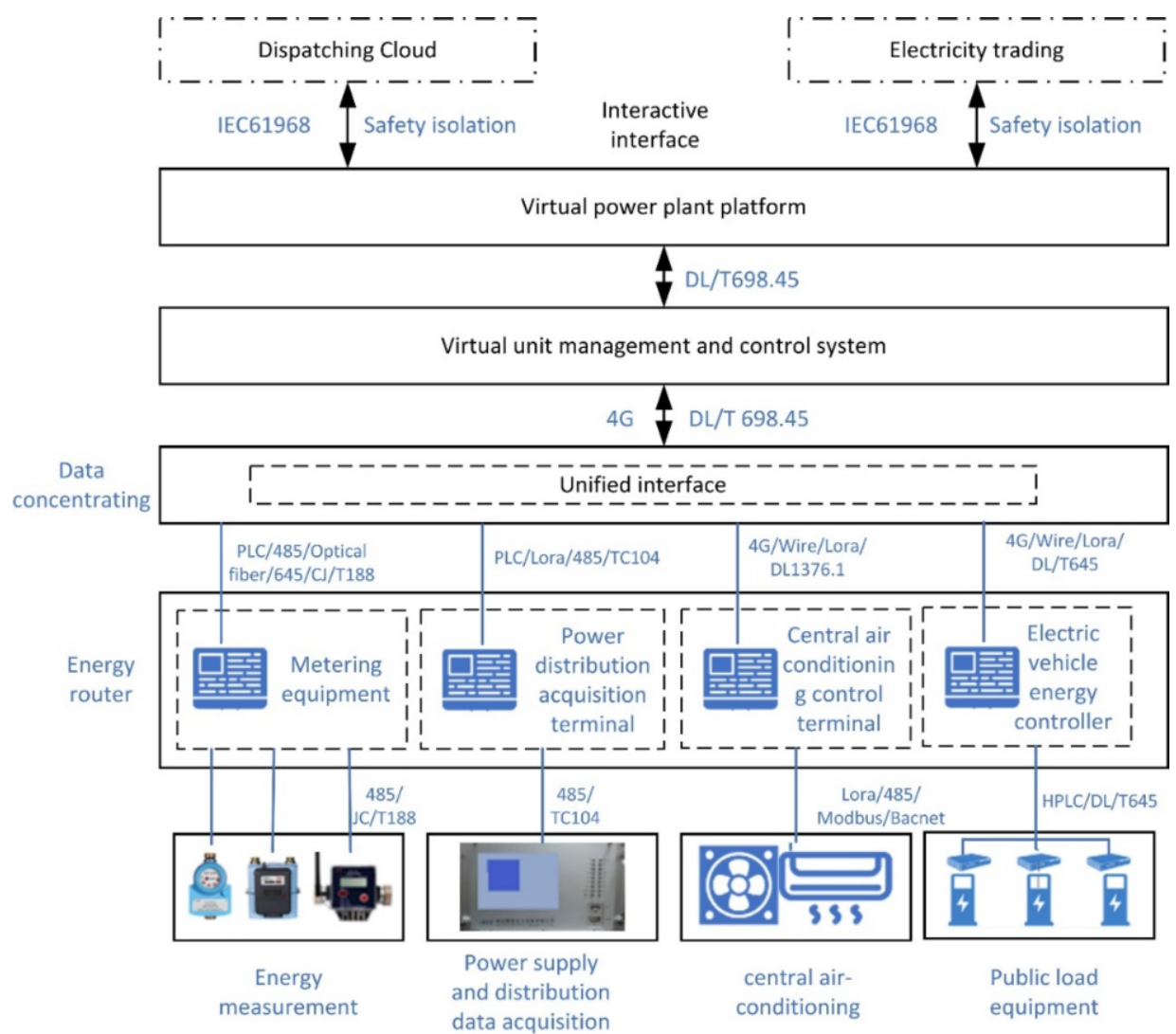

Figure 7. Communication architecture of VPP.

\section{Security Architecture}

The VPP operation management platform is deployed in the external network (not in utility's network). According to the general network security requirements of Chinese national standards and electric power industry standards, the security protection scheme focuses on strengthening the protection design of terminal and virtual unit management and control system security, network boundary security and system security, so as to ensure the safe, reliable and stable operation of the system to the maximum extent.

In the aspect of system security protection, we focus on the host operating system security reinforcement, baseline compliance verification, deployment of anti-virus and anti-malicious code program; In the aspect of network boundary security protection, we focus on communication data encryption and network traffic security monitoring; In the aspect of security protection of virtual unit management and control system, we focus on Ontology security and top-down access security; In terms of terminal and local network security protection, we focus on the identity authentication of energy consuming equipment and lightweight encryption of control strategy issued by virtual unit management and control system. 


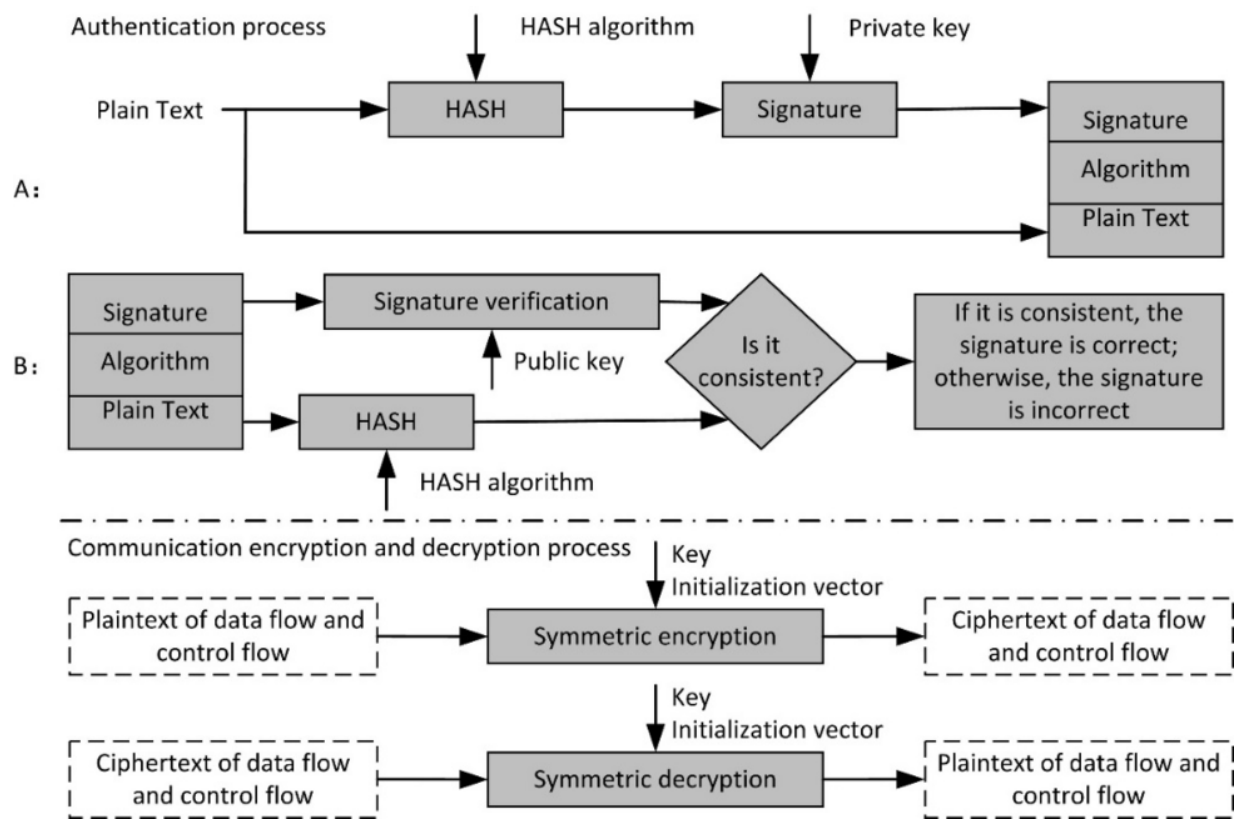

Figure 8. Communication encryption and identity authentication.

Message encryption and identity authentication are very important for the security of VPP platform. As shown in Figure 8, identity authentication is completed based on digital signature and signature verification: A generates fixed length (32 bytes) data for a piece of plaintext data through hash algorithm, and obtains the signature value by signing the fixed length data through private key; B receives the signature value and the plaintext data, uses A's public key to verify the signature value, and performs the same hash operation on the plaintext data, and compares whether the verified data and the hash value are consistent. If they are consistent, the identity of the other party is verified.

The encryption and decryption of communication message is based on symmetric cipher algorithm: the key and initialization vector IV are used for data flow and control flow, and the ciphertext data of data flow and control flow are obtained through symmetric encryption (API interface). Using the same key and initialization vector IV, the original data stream and control stream can be obtained by symmetric decryption (API interface).

The requirements for system data encryption include: the key business data of the system is encrypted and stored; The key business data of the system are transmitted between the systems by encryption and HTTPS; The database connection user has the minimum authority, and the database user password is changed regularly; The database connection information is not exposed to the application system, and the database user password in the application system configuration file must be encrypted; SQL statements are not allowed to be exposed to the client to prevent SQL injection.

\section{Case Study}

We built a virtual power plant pilot project. The project has 36 distributed photovoltaic power stations, 10 energy storage power stations and 22 controllable loads. On this VPP 
software platform, the functions of demand response, energy monitoring, energy analysis, energy optimization and distributed generation trading simulation are realized.

Compared with the traditional demand response software platform, this platform can aggregate a large number of controllable loads which cannot be directly controlled before, with high automation level and fast response; Compared with the same type of foreign VPP platform, the foreign VPP platform focuses on optimizing the generation plan and power consumption plan of distributed generation, while this platform focuses on the control of controllable load. In addition, due to the different rules of China's power market, the functional design of the power trading module of this platform is different from that of the same type of foreign platforms.

\section{Conclusion}

Through the design and implementation of the virtual power plant platform for demand response, we demonstrated the availability and compatibility of virtual power plant technology in China's power system, and accumulated engineering experience. This shows that the scalability, high availability, fast development and high security of the virtual power plant platform in China's power system can be achieved by using microservice architecture, Internet of Things technology, and communication and security technology complying with Chinese standards, so as to meet the requirements of demand response business. Practice has proved that virtual power plant is a beneficial and powerful technical tool to implement demand response.

In order to realize the practicability and commercialization of this platform, our follow-up work and research are as follows:

-Based on the requirements of microservice architecture, various types of distributed resources are modeled;

-Prepare to use domain driven design method, unify requirement analysis and design programming, and design advanced application function module with Python language;

-Research the big data mining technology included association analysis and cluster analysis and so on, and apply it to the software;

-research the power transaction algorithm using blockchain technology to realize peer-to-peer transaction, and uses it to compile the transaction mechanism and bidding platform module of VPP platform;

-research the distributed optimization algorithm based on leader-follower consistency of Multi-Agent System, and the distributed communication, data collection, information exchange and state coordination among agent units are implemented.

\section{References}

[1] Ai Q. Virtual power plants: the ultimate configuration of the energy internet (Science Press). 2018. p11.

[2] Yao JG, Yang SC, Wang K. Power grid dispatching control in the interactive environment of "SourceNetwork-Load" (China Electric Power Press). 2019. p28.

[3] Gao CW, Li QY, Li HX, Zhai HB. Methodology and operation mechanism of demand response resources integration based on load aggregator. Automation of Electric Power Systems. 2013, 37 (17).

[4] Han T, Jiang JD. Source-Network-Load-Storage coordinated control system based on distribution IOT cloud platform and virtual power plant. 5th International Conference on New Energy and Future Energy System. 2020, Xi'an, China. 
[5] Jiang HY, Tan ZF and Hu QH. Action analysis of virtual power plants on energy saving and emission controlling of power industry. Electric Power. 2010, 43 (6).

[6] Huang D, Jiang GD, Feng RQ. Smart park integrated management cloud platform architecture based on microservice governance framework. 4th International Conference on New Energy and Future Energy System, 2019, Macau, China.

[7] Xie CS, Zhong PY. Application system of generic virtual power plant based on service-oriented architecture. East China Electric Power. 2013, 41(8).

[8] Mao T, Guo XX, Xie PP, Zhou J, Zhou BR, Han S. Virtual power plant platforms and their applications in practice: a brief review. 2nd IEEE Sustainable Power and Energy Conference, 2020, Chengdu, China.

[9] Wang Y, Ai X, Tan ZF, Yan L. Interactive dispatch modes and Bidding strategy of multiple virtual power plants based on demand response and game theory. IEEE Transactions on Smart Grid. 2016, 7 (1).

[10] Li X Y, Zhao DM. Multi-level optimal configuration of virtual power plant considering dynamic characteristics of adjustable resources. Automation of Electric Power Systems. 2020, 44(13):17-24.

[11] Manuel W, Sven L, Albert Z. Experience report: first steps towards a microservice architecture for virtual power plants in the energy sector. Microservices. 2019, 2:1-2:10. 